\title{
La Influencia de la Altura de la Capa Límite Oceánica en la Región del Centro de Lanzamientos de Alcántara en Brasil
}

\author{
Luciana B. Pires ${ }^{1}$, Leandro F. Souza ${ }^{2}$, Gilberto Fisch $^{3}$ y Ralf Gielow ${ }^{1}$ \\ (1) Instituto Nacional de Pesquisas Espaciais (INPE), Centro de Previsão do Tempo e Estudos \\ Climáticos (CPTEC), São José dos Campos, SP-Brasil \\ (e-mail:Iuciana.bassi@cptec.inpe.br, ralf.gielow@cptec.inpe.br) \\ (2) Universidade de São Paulo (USP), Instituto de Ciências Matemáticas e de Computação (ICMC), \\ São Carlos, SP-Brasil (e-mail: lefraso@icmc.usp.br) \\ (3) Centro Técnico Aeroespacial (CTA), Instituto de Aeronáutica e Espaço (IAE), \\ São José dos Campos, SP-Brasil (e-mail: gfisch@iae.cta.br)
}

\begin{abstract}
Resumen
Se estudió la influencia de la altura de la capa límite oceánica (CLO) en la región del centro de lanzamiento de cohetes de Alcántara, en Brasil. La región es geográficamente compleja y está constituida de un acantilado con un desnivel de $50 \mathrm{~m}$. Un programa de simulación de flujo 2D fue utilizado en el estudio, con una formulación de vorticidad-velocidad. Se utilizó técnicas compactas de diferencias finitas de alto orden para la discretización del espacio, y la integración en el tiempo fue realizada por el método de Runge-Kutta de cuarto orden. El acantilado fue especificado con el método de fronteras sumergidas. Los resultados numéricos demostraron que la altura de la capa límite oceánica tiene influencia en la formación de la altura de capa limite interna del acantilado, aumentando así la turbulencia en la región.
\end{abstract} interna

\section{The Influence of the Height of the Ocean Boundary Layer in the Region of the Alcântara Space Center in Brazil}

\begin{abstract}
The influence of the height of the ocean boundary layer in the region where the Alcântara Space Center is located, was studied. The topography of the region is very complex and it includes a $50 \mathrm{~m}$ coastal cliff. A 2D numerical code was used, where the equations were written using the vorticityvelocity formulation. High order compact finite differences schemes were used for the spatial derivatives and the time integration was performed with a fourth-order Runge-Kutta scheme. The coastal cliff was specified through the immersed boundary method. The numerical results showed that the height of the ocean boundary layer influences the formation of the height of the internal boundary layer of the cliff, increasing the turbulence in the region.
\end{abstract}

Keywords: Boundary Layer, immersed boundary method, finite differences, internal boundary layer 


\section{INTRODUCCIÓN}

El régimen de vientos y la turbulencia atmosférica en la capa límite superficial han sido objeto de gran interés de la meteorología aeroespacial, tanto para el proyecto y desarrollo de cohetes, como para su lanzamiento, incluyendo la determinación de su trayectoria, o el análisis de las condiciones ambientales durante la ocurrencia de fallas como, por ejemplo, la explosión del Challenger (Uccellini et al., 1986, Fichtl et al., 1988) y el trabajo Kingwell et al. (1991), sobre los factores climáticos que afectan la operación de cohetes.

Johnson (1993), por ejemplo, hace una compilación completa en los elementos climáticos principales de los campos de lanzamiento de cohetes en los Estados Unidos. Kwon et al. (2003), realizaron experimentos en un túnel de viento para estudiar las condiciones atmosféricas de la isla de OenaroDe, donde está el centro del espacio de la Corea del sur (Naro Space Center) en construcción. El conocimiento de la estructura vertical del viento (los perfiles y las ráfagas medias) es muy importante. Por lo tanto, los cohetes son proyectados y se construyen considerando que deben soportar una carga existente por la acción del viento. La trayectoria, el control y dirección de estos son determinados por el perfil del viento siguiente a la superficie. Hasta la altitud de $1000 \mathrm{~m}, 88 \%$ de las correcciones en la trayectoria se deben al viento, mientras que sobre los $5000 \mathrm{~m}$, el viento representa solamente el 3\% de las correcciones (Fisch, 1999).

La región del Centro de Lanzamiento de Alcântara (CLA), de donde se lanzan los cohetes brasileños, presenta a $150 \mathrm{~m}$ de distancia, contra el viento, un acantilado de $50 \mathrm{~m}$ de altura (Fig. 1). Así, el viento, inicialmente en equilibrio con la superficie oceánica lisa, obra recíprocamente con la vegetación arbustiva, localizada en dirección al viento en relación a la cuesta, de la altura media igual los $3 \mathrm{~m}$, formando una Capa de Límite Interna (CLI) dentro de la capa límite atmosférica (Fisch, 1999), debido a la discontinuidad en la superficie generada por el paso del acantilado y por la alteración repentina de la rugosidad entre el océano y el continente (Jegede y Foken, 1998).

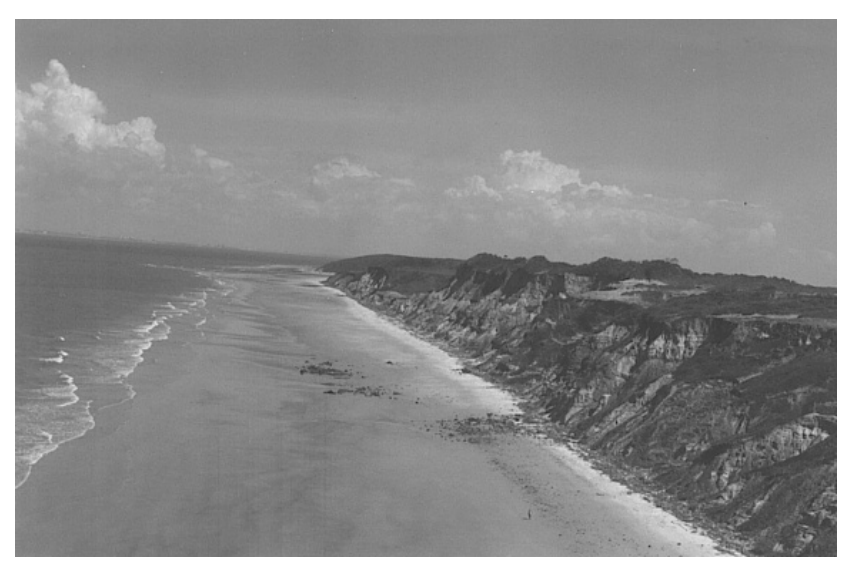

Fig. 1: Acantilado del Centro de Lanzamientos de Alcântara (CLA).

Existe un estudio sobre la rugosidad realizado en la CLA por Roballo y Fisch (2008), analizando los datos del viento recogidos por una torre anemométrica que se encuentra a $200 \mathrm{~m}$ adelante del acantilado, en los períodos 1995 a 1999 y de 2004 a 2005. Las variables consideradas, además de la velocidad del viento local, fueron la velocidad de fricción $\left(u_{*}\right)$ y el largo de rugosidad $\left(z_{0}\right)$, que conforman el perfil logarítmico del viento, el exponente $\alpha$ de la ley de potencia eólica. La velocidad de fricción $u_{*}$ para la temporada de lluvias es igual a $0,32 \pm 0,13 \mathrm{~m} / \mathrm{s}$ y para el seco $0,46 \pm 0,11 \mathrm{~m} / \mathrm{s}$, mientras que $z_{0}$ fue igual a $0,19 \pm 0,32 \mathrm{~m}$ en la estación de las lluvias, y igual a 0,06 $\pm 0,05 \mathrm{~m}$ para el seco. Por lo tanto, el perfil logarítmico del viento es estacional. El exponente $\alpha$ de la ley de potencia para la velocidad osciló entre 0,19 a 0,27 durante los meses del año, mostrando disminución con la altura de $z_{n}$.

Reuter et al. (2004), realizaron un estudio observacional de la capa límite del Océano (CLO) en región del CLA. Los datos se obtuvieron a partir de la liberación simultánea de la radio-sonda del buque Oceanográfico Antares y de la CLA entre los horarios de 12:00 UTC y las 24:00 UTC, durante 
el período del 13 al 15/06/1999. Se observó que la dirección más frecuente es E para la CLA y SE para el océano. La estructura termodinámica media de las atmósferas del océano y de la cuesta fue calculada junto con las características de los elementos meteorológicos en la superficie. El mayor desarrollo de la capa límite convectiva (CLC) costera, en promedio, se produce a las 18:00 UTC (altura de $650 \mathrm{~m}$ ) y en el océano a las 21:00 UTC (altura de $600 \mathrm{~m}$ ). La CLC en la CLA es más cálido $(1,6 \mathrm{~K})$ y húmeda $\left(1,6 \mathrm{~g} \mathrm{~kg}^{-1}\right)$ que sobre el océano. Se observa que la CLC oceánica es más baja que la CLC costera y mantiene su estructura termodinámica por más largo tiempo, hasta las 21:00 UTC, debido a la CLC del océano responder más rápidamente al ciclo diurno de la radiación solar.

El objetivo de este estudio es el análisis de la influencia de la altura de la CLO en la generación de la turbulencia en la CLA, a través de estudios de la vorticidad, de los perfiles de viento y la formación de la CLI, considerándose solamente la CLI neutra, por lo tanto las medias horarias de las velocidades del viento en el CLA son mayores que $10 \mathrm{~m} / \mathrm{s}$, lo qué inhibe los efectos termales, según lo determinado por Fisch (2003).

\section{METODOLOGÍA}

La influencia de la superficie sobre la corriente depende no sólo de las características de este, sino también de las diferencias con respecto a la superficie anterior, cuando el flujo esta en equilibrio. Por lo tanto, una nueva capa de equilibrio es formada donde su espesor vertical aumenta a medida que crece la distancia desde el punto de la discontinuidad. Sobre esta altura, el perfil de viento se encuentra en equilibrio con la superficie anterior y, dentro de esta capa, el perfil del viento se ajusta en relación a la nueva superficie (Stull, 1988). La Figura 2 describe el desarrollo de la CLI y el perfil del viento en las zonas oceánicas $\left(x_{0}\right)$ y continentales ( $x_{1}$ y $\left.x_{2}\right)$, como ocurre en la CLA (Fisch, 1999).

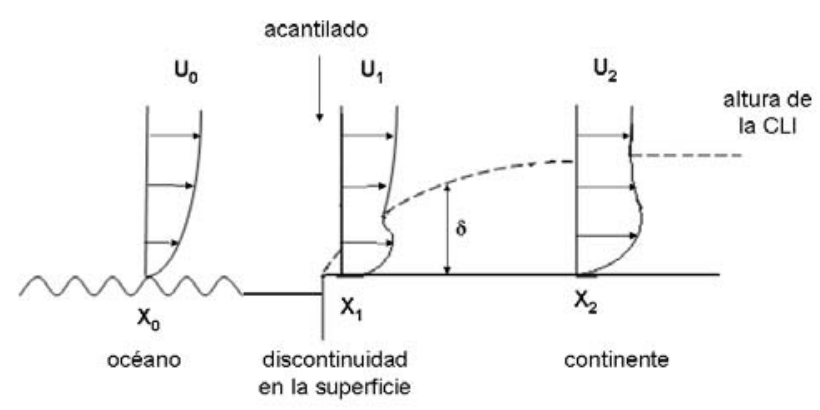

Fig. 2: Representación esquemática del desarrollo del CLI y del perfil del viento en las áreas oceánicas $\left(\mathrm{x}_{0}\right)$ y continentales ( $\mathrm{x}_{1}$ y $\mathrm{x}_{2}$ ). (Fuente: Adaptado de Fisch, 1999).

Savelyev y Taylor (2005), presentaron un compendio de fórmulas para el CLI y una nueva fórmula para predecir la altura de la región de flujo adiabático afectado por un cambio en las condiciones de superficie que se obtuvo como una extensión del caso de corriente neutra. Källstrand y Smedman (1997), presentaron una comparación entre medidas hechas de un avión por toda el área sur de la cuesta este de Suecia y distintos modelos simples para estimar el espesor de la CLI, concluyendo que la mayoría de los modelos sobrestiman el espeso de la CLI. Los modelos proporcionan buenos resultados cerca de la cuesta, pero no a largas distancias (cerca de $15 \mathrm{~km}$ ).

Se adoptó la técnica DNS, resolviendo las ecuaciones numéricamente, usando (i) un integrador para el tiempo del tipo Runge-Kutta de $4^{\circ}$ orden de precisión de almacenamiento bajo y (ii) un método de diferencias finitas compactas de $6^{\circ}$ orden de precisión en las direcciones $x$ y $y$, siendo éste un factor básico y determinativo en la calidad de los resultados. Finalmente, el acantilado fue especificado con el método de fronteras sumergidas. Los resultados de las simulaciones fueron comparados con las observaciones de campaña ECLICA (Pires et al., 2006).

El método de las fronteras sumergidas fue introducido por Peskin (1972), para un flujo incompresible en una región, con los cuerpos sumergidos, que se mueve y fuerza ejercida en ellos. Su ventaja principal es que las ecuaciones de Navier Stokes son resueltas en un dominio rectangular, formando el efecto del interfaz líquido-sólido como una fuerza adicional determinado por la configuración del sólido limitante del flujo, pero manteniendo la precisión y la eficacia numéricas (Souza, 2005). 
El centro de lanzamiento de Alcântara (CLA), está situado en la cuesta del Maranhão ( $2^{\circ} 19^{\prime}$ S; $44^{\circ} 22^{\prime}$ W - altitud de $50 \mathrm{~m}$ ) donde son lanzados los cohetes brasileños como, por ejemplo, el Vehiculo Lanzador de Satélite (VLS) y de Sondaje (SONDA II, SONDA III, VS30 y VS40). La Figura 3 muestra una visión general del CLA. La vegetación en la región del CLA es característica de la región de "restinga", con la altura media alrededor de 2.0 hasta $3.0 \mathrm{~m}$. Su clima presenta un régimen de precipitación dividido en dos períodos: lluvias intensas durante los meses de enero hasta junio, siendo los meses de la marzo y abril los más lluviosos, con un total mensual superior $300 \mathrm{~mm}$; en período seco de julio hasta diciembre, con precipitación inferior a $15 \mathrm{~mm}$ por mes (Fisch, 1999). Los vientos presentan un comportamiento distinto entre las épocas sequías y lluviosas. Durante la estación lluviosa, predomina el viento este hasta aproximadamente altitud de $5000 \mathrm{~m}$, con velocidades alrededor de $7.0-8.0 \mathrm{~m} \mathrm{~s}^{-1}$ en los niveles entre 1000 y $3000 \mathrm{~m}$. En la estación seca, predomina el viento este hasta aproximadamente $8000 \mathrm{~m}$ altitud, con velocidad alrededor de 7.0 $9.0 \mathrm{~m} \mathrm{~s}^{-1}$, siendo intenso en la capa hasta $2000 \mathrm{~m}$, con promedios entre 10.0 y $10.5 \mathrm{~m} \mathrm{~s}^{-1}$, presentando una rotación pequeña hacia el sureste. Esto ocurre por la intensificación de la brisa marítima, que presenta su influencia máxima (contraste termal océano-continente) durante este tiempo, particularmente en los meses de septiembre hasta noviembre (Fisch, 1999). La temperatura del aire y la humedad relativa no presentan variaciones estacionales y sus valores son típicos de la atmósfera tropical, con valores medios mensuales de temperatura entre 26,7 grados $C$ (marzo) y 28,1 grados C (de noviembre) y la humedad relativa entre $77 \%$ (marzo y abril) y el $89 \%$ (octubre y noviembre).

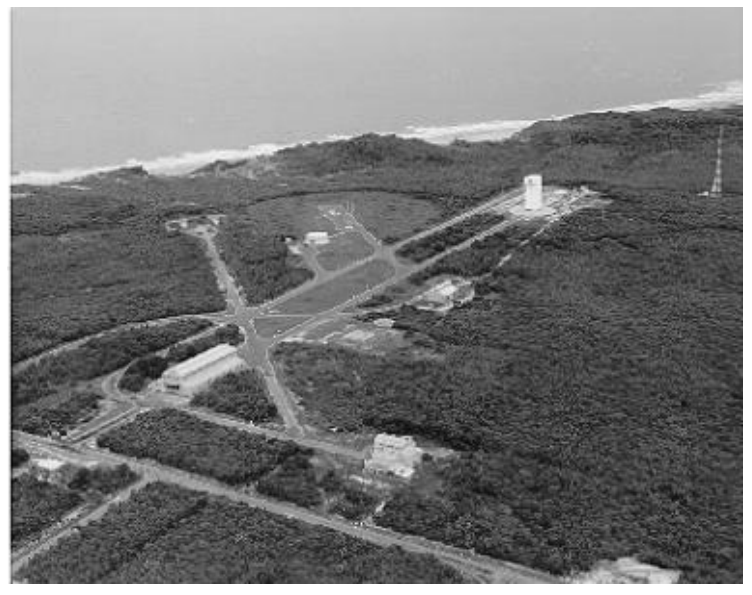

Fig. 3: Visión General del CLA

\section{Formulación}

Las ecuaciones de Navier-Stokes 2D, para el flujo newtoniano, incompresible, con masa específica y viscosidad constantes, con componentes de la velocidad $u$ y $v$, respectivamente en la dirección del flujo $(x)$ y la dirección vertical $(y)$, incorporando la condición de la frontera sumergida que se representa por las fuerzas $F_{x}$ y $F_{y}$ (Góis y Souza, 2007), y se expresan en coordenadas cartesianos ortogonales, siendo $P$ la presión, se convierten en:

$$
\begin{aligned}
& \frac{\partial u}{\partial t}+u \frac{\partial u}{\partial x}+v \frac{\partial u}{\partial y}=-\frac{\partial P}{\partial x}+\frac{1}{\operatorname{Re}} \nabla^{2} u+F_{x} \\
& \frac{\partial v}{\partial t}+u \frac{\partial v}{\partial x}+v \frac{\partial v}{\partial y}=-\frac{\partial P}{\partial y}+\frac{1}{\operatorname{Re}} \nabla^{2} v+F_{y}
\end{aligned}
$$

con la ecuación de la continuidad:

$$
\frac{\partial u}{\partial x}+\frac{\partial v}{\partial y}=0
$$

Las variables usadas en las ecuaciones anteriores son adimensionales y se relacionan con las dimensionales a través: 
$x=\frac{\bar{x}}{\bar{L}}, y=\frac{\bar{y}}{\bar{L}}, u=\frac{\bar{u}}{\bar{U}_{\infty}}, v=\frac{\bar{v}}{\bar{U}_{\infty}}, t=\frac{\bar{t} \bar{U}_{\infty}}{\bar{L}}, \operatorname{Re}=\frac{\bar{U}_{\infty} \bar{L}}{\bar{v}}$

donde, Re es el número de Reynolds y los términos con una barra superior son dimensionales: $\bar{L}$ es el largo del paso, $\bar{U}_{\infty}$ es la velocidad encima de CLI y $\bar{v}$ es la viscosidad cinemática. La ecuación del transporte del vorticidad, $\omega_{z}$, se consigue de la aplicación del operador rotatorio en las ecuaciones del momentum, en las Eqs. (1) y (2), resultando:

$\frac{\partial \omega_{z}}{\partial t}=-u \frac{\partial \omega_{z}}{\partial x}-v \frac{\partial \omega_{z}}{\partial y}+\nabla^{2} \omega_{z}+\frac{\partial F_{x}}{\partial y}-\frac{\partial F_{y}}{\partial x}$

Utilizando la definición de verticidad y la ecuación de la continuidad, se tiene una ecuación de Poisson para la componente $v$, como:

$\frac{\partial^{2} v}{\partial x^{2}}+\frac{\partial^{2} v}{\partial y^{2}}=-\frac{\partial \omega_{z}}{\partial x}$

Primero es encontrada la solución de Blasius (sin paso) y, que es mi perfil de velocidad en la entrada para un $\operatorname{Re}=3 \times 10^{6}$, enseguida, se introduce la condición de frontera sumergida (paso) y se resuelve el problema completo.

\section{Método numérico}

El código fue desarrollado en el Instituto de Ciencias Matemáticas y Computación (ICMC) de la Universidad de São Paulo (USP),campus de São Carlos, y se adaptó a la situación de los acantilados costeros. La computadora usada fue un modelo AMD 64X2 4400+ con 3Gb de memoria ram cada una. El tiempo total de simulación fue entre 72 y 120 horas, mayor con el aumento de Re. En la malla utilizada habían 801 puntos en la dirección x y 305 en la dirección y. Se adoptó el $\Delta t$ de forma a que el CFL fuera alrededor del 0,2. Las condiciones de contorno son, en la entrada del dominio de integración $\left(x=x_{0}\right)$, las componentes de la velocidad $y$ el vorticidad son fijas. En la salida $\left(x=x_{\max }\right)$, son nuas las segundas derivadas de las componentes de velocidad y de vorticidad en la dirección del flujo. En el límite superior $\left(y=y_{\max }\right.$ ) las derivadas de $v$ en la dirección vertical y son nulas, y en el inferior $(y=0), u$ y $v$ son nulas, quedando la verticidad definida por:

$$
\frac{\partial \omega}{\partial x}=-\frac{\partial^{2} v}{\partial y^{2}}
$$

Una zona de amortiguación fue utilizada en las simulaciones para forzar los disturbios a bajar gradualmente a cero después del dominio en la dirección del flujo, $x$, de interés. La idea básica es multiplicar los componentes del vorticidad para una función de rampa $f 2(x)$, después de cada paso de la técnica de integración. Esta técnica fue probada por Kloker (1998), como muy eficiente en la prevención de las reflexiones que vienen de contornos en la simulación de flujos con disturbios en las propagaciones. Usando esta técnica, los componentes de la vorticidad se dan por:

$\omega_{z}(x, y)=f_{2}(x) \omega(x, y, t)$

donde $\omega(x, y, t)$ es el componente de la vorticidad que viene de la técnica de la integración temporal, $y$ $f 2(x)$ es una función rampa que varía suavemente de 1 hasta 0 . La función puesta en ejecución en la dirección $x$ es:

$f_{2}(x)=f(\epsilon)=1-6 \epsilon^{5}+15 \epsilon^{4}-10 \epsilon^{3}$

con $\in=\frac{\left(i-i_{3}\right)}{i_{4}-i_{3}}$ para $i_{3} \leq i \leq i_{4}$ correspondientes a las posiciones $x_{3}$ y $x_{4}$ en la dirección del flujo, respectivamente. Para asegurar un buen resultado numérico, una distancia mínima entre $x_{4}$ y el extremo del dominio $x_{\max }$ debe ser especificada. 
Las derivados del espacio fueron calculadas usando una técnica de diferencias finitas compactas de $6^{\circ}$ orden (Souza et al., 2005). La ecuación de Poisson fue resuelta usando una técnica de multigrid de acercamiento completo en ciclo-v de integración, trabajando con 5 grids.

Los valores de las fuerzas en el contorno habían sido calculados usando las ecuaciones siguientes:

$F_{x}(i, j)=\delta f r\left(\right.$ vel $\left._{u}\right)$
$F_{y}(i, j)=\delta f r\left(\right.$ vel $\left._{v}\right)$

donde $f r$ es el término de relajación igual a - Re y $\delta$ un valor que sea cero fuera de la frontera sumergida y uno dentro de ella. Para la interacción temporal fue utilizada la técnica de Runge-Kutta de $4^{\circ}$ orden de memoria reducida. El tiempo de evolución de la ecuación puede ser escrita como (Santana y Souza, 2006):

$\frac{\partial U}{\partial t}=F(U)$

donde $U$ representa el vector que contiene los valores de la solución de las funciones espaciales y de los derivados en diferencias finitas. Una técnica explícita de Runge-Kutta de memoria reducida, está orientada en la solución del tiempo $t_{n}$ para $t_{n}+\Delta t$ en la forma:

$w^{0}=w^{n}$
$w^{\prime}=w^{0}+\frac{\Delta t}{4} w^{\prime 0}$,
$w^{2}=w^{0}+\frac{\Delta t}{3} w^{\prime 1}$
$w^{3}=w^{0}+\frac{\Delta t}{2} w^{\prime 2}$
$w^{n+\Delta t}=w^{4}=w^{0}+\Delta t w^{\prime 3}$

\section{RESULTADOS Y DISCUSIÓN}

El la campaña de observación ECLICA, realizada en el período de 6 hasta 16 de octubre de 1998, se determinó que la altura de la CLI, distante $50 \mathrm{~m}$ del acantilado en dirección del flujo, esta entre 4.5 y $9 \mathrm{~m}$, y es superior a $15 \mathrm{~m}$ de altura a $100 \mathrm{~m}$ del acantilado (Pires et al., 2006). El número de Reynolds atmosférico representativo del CLA es $3 \times 10^{7}$, con base en la altura del acantilado. Sin embargo, en este trabajo $3 \times 10^{6}$ fue utilizado, por razón del tiempo de cómputo y la representación física, ya que para los casos de orden de $10^{7}$, los resultados numéricos llegan a ser no estáticos, con despliegue sucesivo de vórtices.

Las Figuras 4 a 7 muestran los campos de la vorticidad formados sobre el acantilado con las CLO's incidentes de 200, 250, 280 y 300 m. Para la CLO de 200 m (Fig. 4), se puede ver la formación de una burbuja con una intensidad de vorticidad de $-100 \mathrm{~s}-1$, justo encima del acantilado, seguido por una vorticidad positiva de $7000 \mathrm{~s}-1$. Nótese, que con el aumento en la altura de la CLO, respectivamente, 250, 280 y 300 m (Figs. 5, 6 y 7), la burbuja de recirculación tiende a ser más extensa y con la formación de una CLI más alta. Pero, se ve que la burbuja de recirculación que alcanza su máximo en $\mathrm{x}=100 \mathrm{~m}$ (Fig. 7), no llega a la plataforma de lanzamiento que se encuentra a 150 metros del acantilado.

Las Figuras 8 a 11 muestran los perfiles de viento en $x=50,100$ y 150 metros, en la parte superior del acantilado. Cabe señalar que en $50 \mathrm{~m}$ (Fig. 8), la CLO de $200 \mathrm{~m}$, tiene un perfil distinto de los demás, siendo negativa a la altura de $55 \mathrm{~m}$, lo que representa la mayor intensidad de la burbuja de recirculación. Los otros perfiles tienen una tendencia positiva, quedando por encima de cero. 


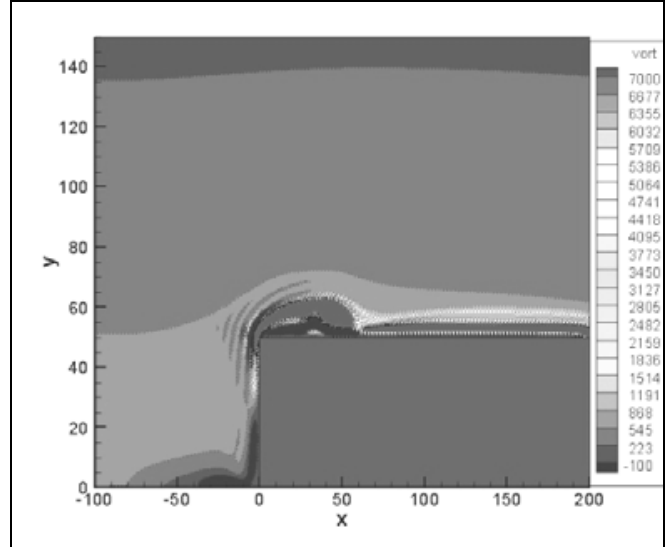

Fig. 4: Contorno de vorticidad para la capa límite oceánica de $200 \mathrm{~m}-\operatorname{Re}=3 \times 10^{6}$.

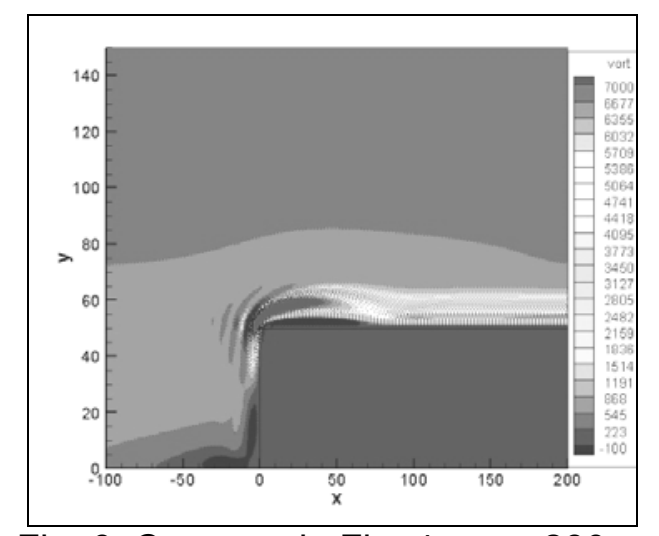

Fig. 6: Como en la Fig. 4, para $280 \mathrm{~m}$

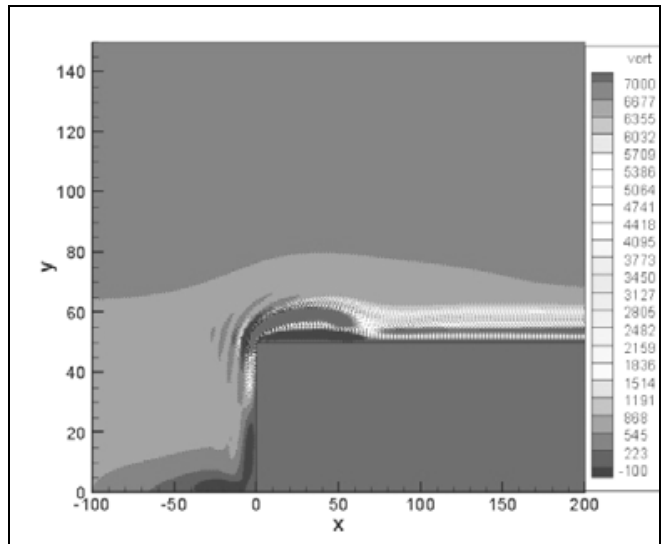

Fig. 5: Como en la Fig. 4, para $250 \mathrm{~m}$

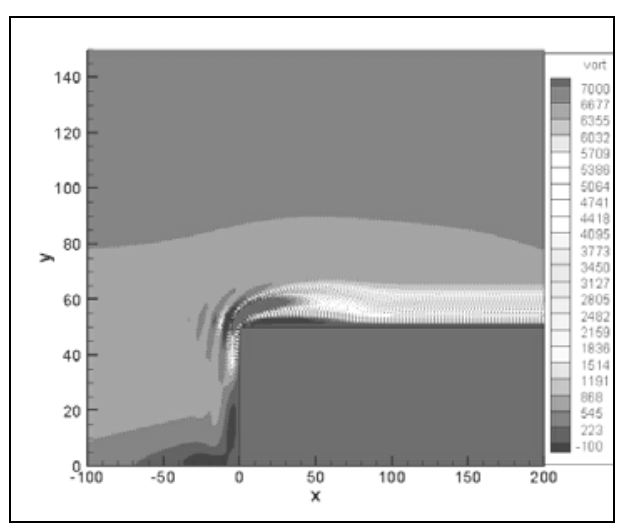

Fig. 7: Como en la Fig. 4, para 300 m

En 100 m (Fig. 9), la burbuja parece disiparse, por otro lado, para el perfil de la CLO de $200 \mathrm{~m}$, tienen un mayor valor de $U$ hasta la altura de $55 \mathrm{~m}$, con el menor valor de U presentado para la CLO de 300 $\mathrm{m}$. Los perfiles a $150 \mathrm{~m}$ (Fig. 10) son similares lo que sugiere que la corriente ya está en equilibrio con la nueva superficie. Cabe señalar que en todos los casos, los perfiles de $U$ demostrado, disminuyen con el aumento de la altura de la CLO. Analizando la distribución de la componente normal de la velocidad en la dirección y (V) en $x=50 \mathrm{~m}$ (Fig. 11) se puede ver el flujo contrario cerca de la superficie hasta los 60 metros de altura, siendo más intenso para la CLO de $200 \mathrm{~m}$. Estos valores negativos para $\mathrm{V}$ ocurren porque en esta posición ya tenemos el final de la burbuja de recirculación. También se ve que, con el crecimiento de la altura de la capa límite, esta burbuja tiende a disminuir en tamaño. Para las CLOs de 280 y 300 no hay casi diferencia entre los perfiles de $\mathrm{V}$. En $\mathrm{x}=100 \mathrm{~m}$ (Fig. 12), tenemos un retroceso en la distribución de esa componente para la CLO de $200 \mathrm{~m}$, que ahora presenta un pico positivo cerca de $60 \mathrm{~m}$ de altura. De acuerdo con la Fig. 13 se ve que la región se disipa cuando se distancia del acantilado, lo que confirma la disipación de la burbuja de recirculación.

La Figura 14 presenta la CLI generada para cada CLO. La altura de la CLI fue determinada por la búsqueda de la posición donde $\partial \mathrm{wz} / \partial \mathrm{y} \approx 0$. Observando la altura de la CLI, arriba del acantilado de $50 \mathrm{~m}$, se ve los siguientes valores: (i) en $\mathrm{x}=50 \mathrm{~m}$, la altura de la CLI es $17 \mathrm{~m}$, para la CLO de 200 m; $18 \mathrm{~m}$ para la CLO de $250 \mathrm{~m} ; 19 \mathrm{~m}$ para la CLO de $280 \mathrm{~m}$ y $20 \mathrm{~m}$ para la CLO de $300 \mathrm{~m}$. (ii) Para la distancia de $\mathrm{x}=100 \mathrm{~m}$ del acantilado, la región de recirculación para la CLO de $200 \mathrm{~m}$ parece tener disminuido e as CLl's presentan los valores de 12, 16, 19 e $21 \mathrm{~m}$, respectivamente, para las CLO's de 200, 250, 280 e 300 m. (iii) Para a distancia de $x=150 \mathrm{~m}$ del acantilado, donde se localiza la rampa de lanzamiento de cohetes, la altura alcanzada por la CLI para las CLO's de 200, 250, 280 e $300 \mathrm{~m}$ son, respectivamente, de: 13, 17, 19 e $21 \mathrm{~m}$. (iv) Los resultados presentados en $\mathrm{x}=200 \mathrm{~m}$ del acantilado, son semejante a los resultados a distancia de $150 \mathrm{~m}$.

Es interesante observar que la configuración varía poco para los valores más grandes de la CLO (CLO's de 280 y 300) lo qué indica un comportamiento asintótico. 


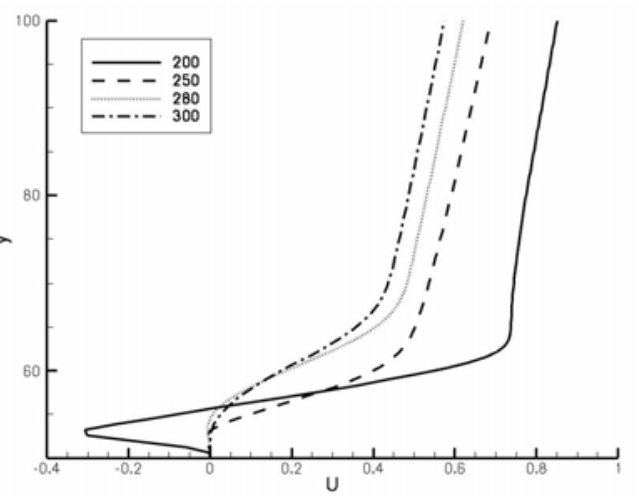

Fig. 8: Perfiles del viento para la capa límite oceánica en $\mathrm{x}=50 \mathrm{~m}$.

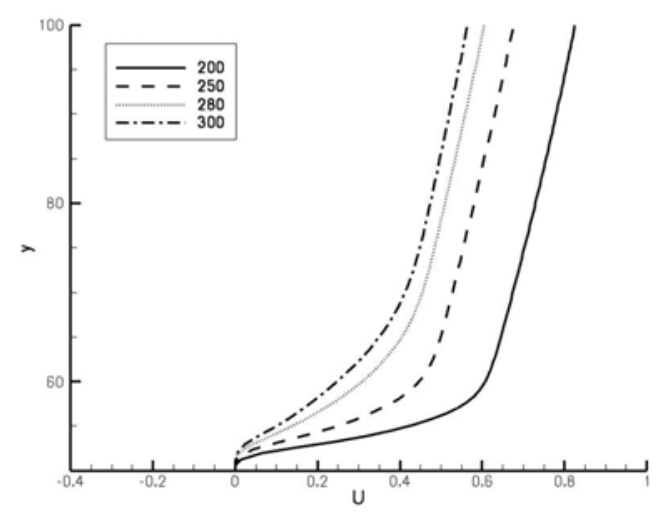

Fig. 10: Perfiles del viento para la capa límite oceánica em $x=150 \mathrm{~m}$.

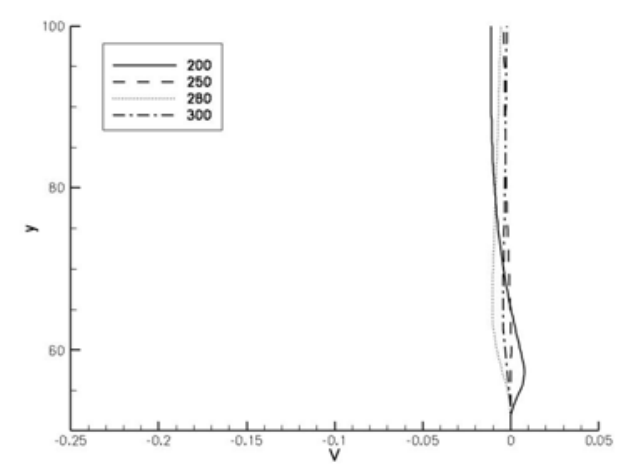

Fig. 12: Distribución de la componente normal de la velocidad en la dirección y para $x=100 \mathrm{~m}$

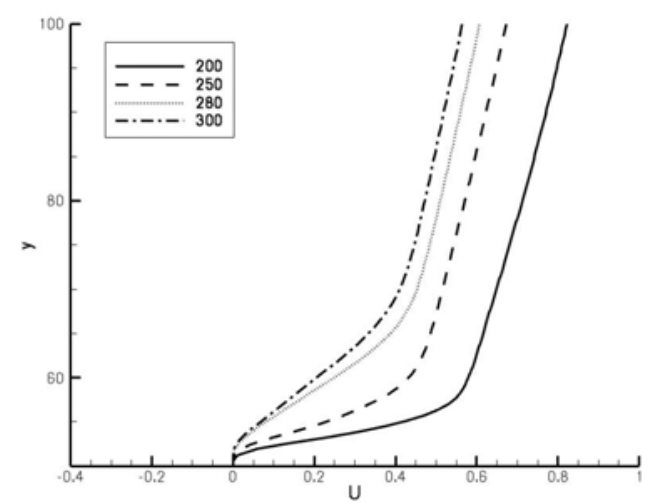

Fig. 9: Perfiles del viento para la capa límite oceánica en $x=100 \mathrm{~m}$.

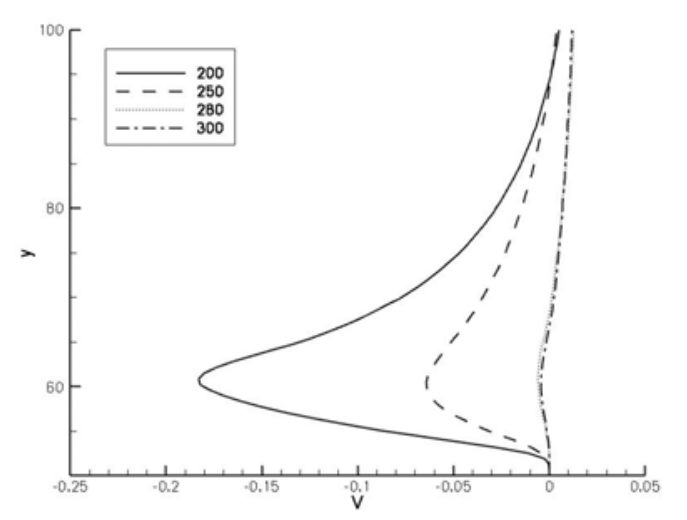

Fig. 11: Distribución de la componente normal de la velocidad en la dirección y para $x=50 \mathrm{~m}$

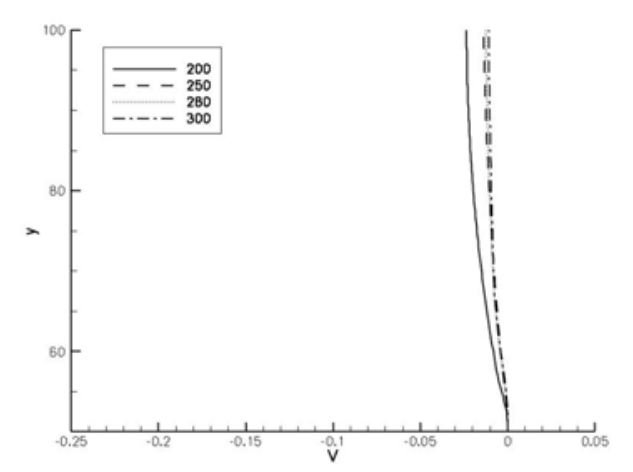

Fig. 13: Distribución de la componente normal de la velocidad en la dirección y para $x=150 \mathrm{~m}$

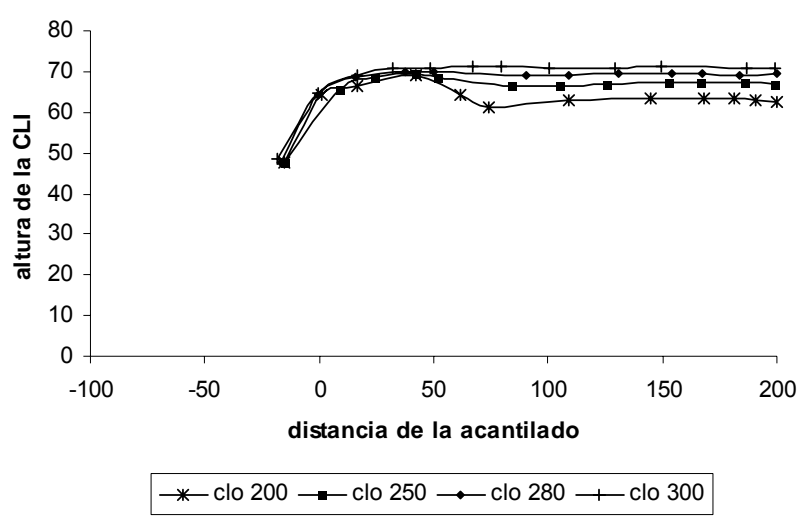

Fig. 14: Crecimiento de la CLI de acuerdo con la CLO 


\section{CONCLUSIONES}

En general, la altura de la Capa Límite Oceánica - CLO influencian la altura del CLI formado en dirección del flujo en relación al acantilado, aumentando así la turbulencia en esta región.

Se observa, que para una CLO más baja, la burbuja de recirculación es más intensa, y al mismo tiempo más corta. Cuanto mayor sea la altura de la CLO, la burbuja es más larga, y mayor es la CLI formada. La CLI numéricamente calculada tiende a un valor asintótico para un número fijo de Reynolds.

Se observó que la región de recirculación no llega a la plataforma de lanzamiento para cohetes. Mientras tanto, la CLI alcanza una altura de 13, 17, 19 y $21 \mathrm{~m}$, respectivamente, para las CLOs de 200, 250, 280 y 300. Como la torre móvil de integración (donde los cohetes se construyen), en la plataforma de lanzamiento, tiene una altura de $20 \mathrm{~m}$, se concluye que él sufre una fuerte influencia de la CLI.

En todos los casos se aprecia una sobreestimación del modelo para los datos más cercano acantilado, en comparación con los datos de observación de campaña ECLICA, donde a 50 metros del acantilado, la altura de la CLI es entre 4.5 y $9 \mathrm{~m}$, pero para datos más distantes que 100 metros del acantilado, hemos obtenido un buen acuerdo.

\section{NOMENCLATURA}

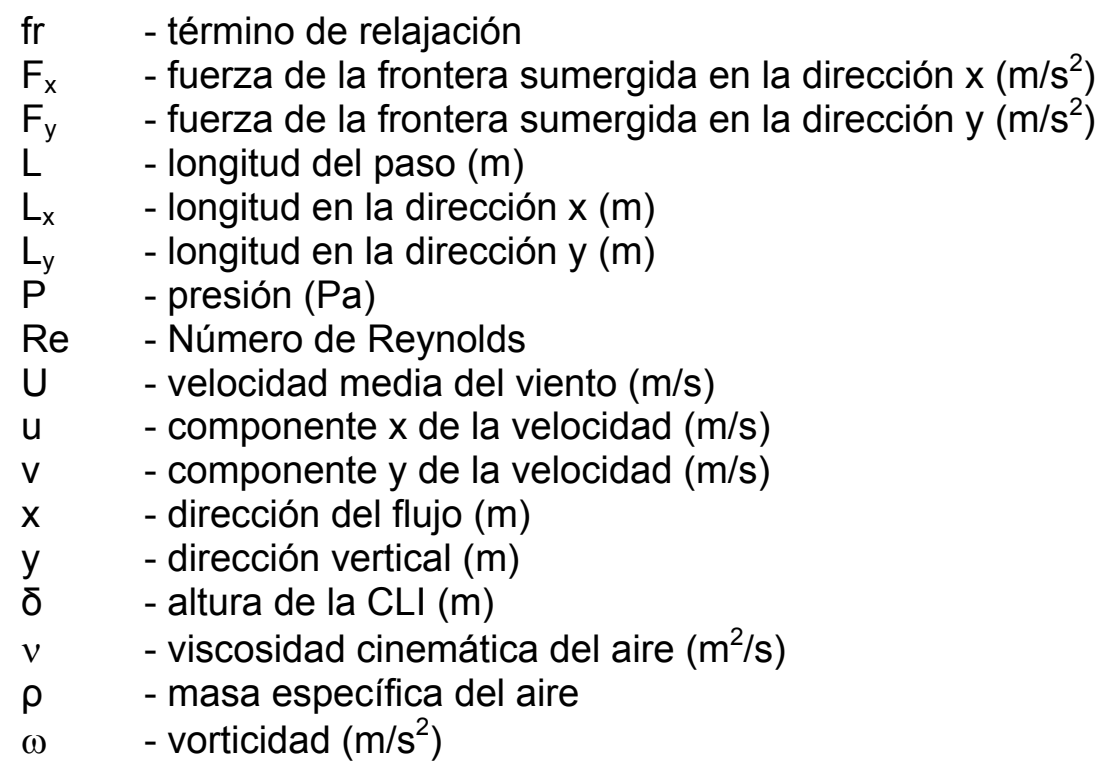

\section{AGRADECIMENTOS}

Los autores agradecen Rafael Cuenca por la traducción. L. B. M. Pires agradece al CNPq por su bolsa de doctorado, y G. Fisch (Proc. 302117/2004-0) y L. F. Souza (Processo 04/16064-9) agradecen al soporte de FAPESP.

\section{REFERENCIAS}

Fichtl, G. H. y otros nueve autores; Analysis of In-Flight Winds for Shuttle Mission STS 51-L, Journal Applied Meteorology: 27(1), 1232-1241 (1988).

Fisch G.; Características do Perfil Vertical do Vento no Centro de Lançamento de Foguetes de Alcântara (CLA), Revista Brasileira de Meteorologia: 14(1), 11-21 (1999).

Fisch, G.; Análise da Turbulência Atmosférica no Centro de Lançamento de Foguetes de Alcântara, Revista Ciência \& Natura: Edição especial, 211-215 (2003). 
Góis, E.R.C. y L.F. Souza; Numerical Study of Lockin Phenomena in 2D Flow over a Cylinder, Proceedings of the XII International Symposium on Dynamic Problems of Mechanics (DINAME 2007), Ilhabela, SP, Brasil 26 de Fevereiro a 2 de Marzo (2007).

Jegede, O.O y T.H. Foken; A Study of the Internal Boundary Layer due to a Roughness Change in Neutral Conditions Observed During the LINEX Field Campaigns, Theoretical and Applied Climatology: 62, 31-41 (1998).

Johnson, D.L.; Terrestrial environment (climatic) criteria guidelines for use in aerospace vehicle development, 1993 revision. NASA TM 4511, Huntsville, AL, USA, 472 p. (1993)

Källstrand B. y A.S. Smedman; A case study of the near-neutral coastal internal boundary-layer growth: aircraft measurements compared with different model estimates. Boundary Layer Meteorology: 85, 1-33 (1997).

Kingwell, J. y otros cuatro autores; Weather Factors Affecting Rocket Operations: A Review and Case History, Bulletin American Meteorological Society: 72(6), 778-793 (1991).

Kloker, M.; A robust high-resolution split-type compact fd scheme for spatial direct numerical simulation of boundary-layer transition, Applied Scientific Research: 59, 353-377 (1998).

Kwon, K.J., J. Y. Lee y B., Sung; PIV Measurements on the boundary Layer Flow around Naro Space Center, 5th International Symposium on Particle Image Velocimetry, Paper 3121, Busan, Corea, 22 a 24 de Septiembre (2003).

Peskin, C.S.; Flow Patterns Around Heart Valves: A Numerical Method, Journal Computational Physics: 10, 252-271 (1972).

Pires, L.B.M., G. Fisch y R. Gielow; Desenvolvimento da Camada Limite Interna no Centro de Lançamento de Alcântara, XIV Congreso Brasileño de Meteorología, Florianópolis, SC, Brasil, 27 de noviembre a 01 de deciembre (2006).

Reuter, E.D.J., G. Fisch, G.V. Mota y J.C.P., Cohen; Estudo observacional da Camada Limite Planetária Marinha na Região do Centro de Lançamento de Foguetes de Alcântara - MA, Revista Brasileira de Meteorologia: 19(3), 251-264 (2004).

Roballo, S.T. y G. Fisch; Escoamento atmosférico no Centro de Lançamento de Alcântara (CLA): parte I - aspectos observacionais, Revista Brasileira de Meteorologia: 23 (3), no prelo (2008).

Santana, L.D. y L.F. Souza; Numerical Solution of 2-Dimensional Linearized Euler Equations for Acoustic Waves in Flow, Proceedings of the 11th Brazilian Congress of Thermal Sciences and Engineering, Curitiba, Brasil, 5 a 8 de Deciembre (2006).

Savelyev S.A. y P.A. Taylor, Internal Boundary Layers: I. Height Formulae for Neutral and Adiabatic Flows, Boundary Layer Meteorology: 115, 1-25 (2005).

Souza, L.F.; Numerical Simulation of a Flow over a Circular Cylinder using Immersed Boundary Method in Vorticity-Velocity Formulation, Procedings of 18th International Congress of Mechanical Engineering, Ouro Preto, MG, Brasil, 6 a 11 de noviembre (2005).

Souza, L.F., M.T. Mendonça y M.A.F. Medeiros; The advantages of using high-order nite differences schemes in laminar-turbulent transition studies, Int. J. for Numer. Meth. in Fluids: 48, 565-592 (2005).

Stull R.; An Introduction to Boundary Layer Meteorology, Kluwer Academic Publ., 666 p, London (1988).

Uccellini, L. W. y otros seis autores; A report on the upper-level wind conditions preceding and during the Shuttle Challenger (STS 51 L) Explosion, Bulletin of the American Meteorological Society: 67(10), 1248-1265 (1986). 